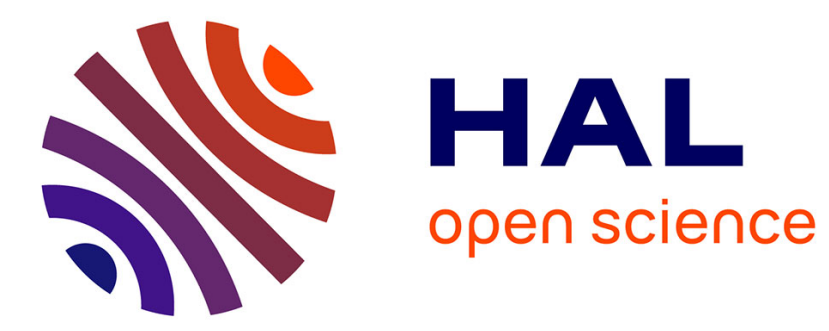

\title{
Deflections of highly inflated fabric tubes
}

Jean-Christophe Thomas, Christian Wielgosz

\section{To cite this version:}

Jean-Christophe Thomas, Christian Wielgosz. Deflections of highly inflated fabric tubes. Thin-Walled Structures, 2004, 42 (7), pp.1049-1066. 10.1016/j.tws.2004.03.007 . hal-01006720

\section{HAL Id: hal-01006720 \\ https://hal.science/hal-01006720}

Submitted on 3 Dec 2016

HAL is a multi-disciplinary open access archive for the deposit and dissemination of scientific research documents, whether they are published or not. The documents may come from teaching and research institutions in France or abroad, or from public or private research centers.
L'archive ouverte pluridisciplinaire HAL, est destinée au dépôt et à la diffusion de documents scientifiques de niveau recherche, publiés ou non, émanant des établissements d'enseignement et de recherche français ou étrangers, des laboratoires publics ou privés. 


\title{
Deflections of highly inflated fabric tubes
}

\author{
J.-C. Thomas, C. Wielgosz
}

GeM-Institut de Recherche en Génie Civil et Mécanique, Faculté des Sciences et des Techniques, Université de Nantes, 2, rue de la Houssinière, BP 92208, 44322 Nantes Cedex 03, France

Inflatable beams made of modern textile materials with important mechanical characteristics can be inflated at high pressure. The aim of the paper is to present experimental, analytical and numerical results on the deflections of highly inflated fabric tubes submitted to bending loads. Experiments are displayed and we show that tube behaviour looks like that of inflatable panels (Thin-Walled Struct. 40 (2002) 523-536). Equilibrium equations are once again written in the deformed state to take into account the geometrical stiffness and the following forces. The influence of the shear stress cannot be neglected and Timoshenko's beam theory is used. A new inflatable tube theory is established and simple analytical formulas are given for a cantilever-inflated tube. Comparisons between analytical and experimental results are shown. A new inflatable finite tube element is constructed by use of algebraic operations, because the compliance matrix of the cantilever beam is not symmetric. Comparisons between experimental, analytical and numerical results prove the accuracy of this beam theory and on this new finite element for solving problems on the deflections of highly inflated tubes.

Keywords: Inflatable tubes; High pressure; Following forces; Inflatable finite tube element

\section{Introduction}

In a recent paper [1], we constructed the mechanical theory of highly inflated panels. Their behaviour depends mainly on the material characteristics of the fabrics, the applied pressure and the external loading. When the pressure is low, panels behave like yarns. At high pressure, they behave like beams and yarns, and when the external load is near their collapse load, their shape looks once again like 
a yarn or mechanism behaviour. In fact, we have proved that their deflections are simply given by the sum of yarn's and beam's theory. The study of inflated tubes presents similarities to those of inflatable panels, but the effects of the shear stresses which have been neglected in the case of panels cannot be avoided for tubes and quite different results are obtained.

The first results on the deflections and collapse loads of cantilever inflatable tubes can be found in Comer and Levy [2]. They have assumed that these structures can be studied by using the usual Bernoulli's beam theory. Fichter [3] has used the minimization of the global potential energy of inflated beams to propound another solution. His result leads the way to a behaviour similar to those of inflatable panels, but needs to be improved for tubes. Main et al. [4] have realized experiments on inflatable tubes and compared their results to Comer's theory. In the last study, the maximum internal pressure of the tubes was about $70 \mathrm{kPa}$ and the characteristics of the material used to make the experiments were plainly lower than those of the fabrics used in this study. Their results on the collapse loads are only relative to cantilever tubes. We have shown [5] that the usual theory of limit analysis of beams can be applied to the computation of collapse loads of hyperstatic inflatable tubes in reserve to an adapted definition of the limit momentum of the inflatable beam. With respect to the deflection analysis, we will show that the usual beam theory cannot be used because the pressure does not appear in the values of the deflections of the tube. It seems clear that deflections of fabric tubes inflated at low pressures can be very large when the applied external load reaches the collapse load of the tube whatever the constitutive law of the fabric. The internal pressure must therefore appear in the formulas giving the deflections of inflated structures.

In the first section of the paper we will describe experimental work and show that Timoshenko's beam theory must once again be used because the tube's sections do not remain orthogonal to the neutral fibre of the tube after deformation. The inflatable tube theory is constructed in the second section of the paper. Equilibrium equations are written in the deformed state to take into account the geometrical stiffness of the beam and the following forces due to the internal pressure. Timoshenko's beam theory is used to describe the kinematics of the tube. The shear stress is taken into account and gives a coupling between yarn and beam effects. Simple analytical formulas are provided for a cantilever-inflated tube submitted to pressures going up to several hundred $\mathrm{kPa}$. Comparisons between these analytical results on the deflections of inflatable tubes at high pressure and experimental ones are displayed. Analytical results on the deflections are only relative to isostatic inflatable beams. The next section of the paper is devoted to construct an inflatable beam finite element able to give accurate values of the displacement field for hyperstatic tubes and also for structures made of inflatable tubes. For inflatable panels, the compliance matrix of a cantilever-inflated panel is nothing but the sum of the yarn and beam compliances. The stiffness matrix depends on the inflation pressure and is simply obtained by the usual theory of the equilibrium FEM [6]. For tube problems, the compliance is not symmetrical. We have chosen to use the "left-quotient operation" defined in [7] to derive a new non-symmetrical finite 
element also dependent on the pressure and which allows the study of tubes in hyperstatic configurations. Comparisons between experimental and numerical results are shown. Numerical results are similar to experimental ones.

\section{Experimental results on inflatable tubes}

The tested tubes are prototypes made of Ferrari's prestressed fabrics. The building process of the fabric is such that Young's moduli in the warp and weft directions are almost the same. Tubes come from a strip of fabric welded at high frequency. Experiments have been done for simply supported tubes loaded by a concentrated force (Fig. 1). Two metallic crowns mounted on ball bearings allow the rotation of two sections of the tube. An PVC cylinder allows to fit the diameter of the inflated tube. One of the crowns can translate with a special guidance system. The shape of the tubes is measured with a high resolution numerical camera.

The shape of the deformed tube is shown Fig. 1. The inflated tubes have been submitted to three levels of pressure: 100, 200 and $300 \mathrm{kPa}$. They have been tested for loads varying until the wrinkle load (when the first wrinkle appears) and for loads varying between the wrinkle load and the collapse load (when the tube does not resist any more). The bending shapes of the tubes seem like those of usual beams and the tubes do not present straight parts like the case of inflated panels. Fig. 2 proves that the usual Bernoulli theory cannot be used because the tube's sections do not remain orthogonal to the neutral fibre of the tube after deformation. Timoshenko's beam theory must therefore be used. Moreover, the inflation pressure does not appear in the usual beam solution [2]. Table 1 shows that the

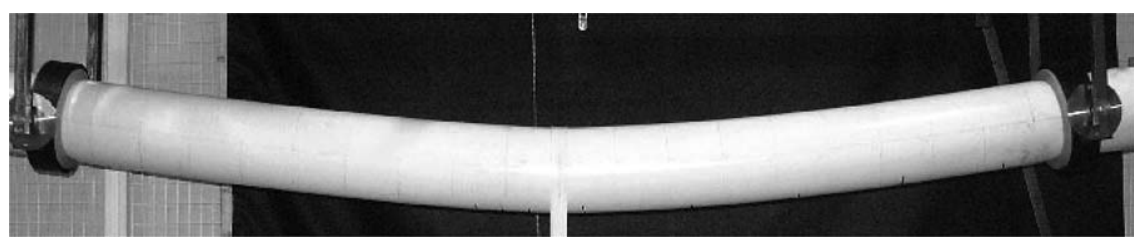

Fig. 1. Deflection of a highly inflated tube $(300 \mathrm{kPa})$.

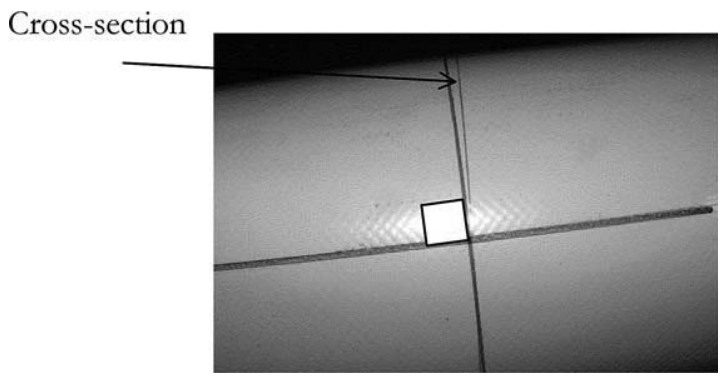

Fig. 2. Rotation of the straight section. 
Table 1

Experimental values of the deflection of the inflated tube $F=147 \mathrm{~N}$

\begin{tabular}{lll}
\hline Pressure & $100 \mathrm{kPa}$ & $300 \mathrm{kPa}$ \\
Deflection & $0.061 \mathrm{~m}$ & $0.030 \mathrm{~m}$ \\
\hline
\end{tabular}

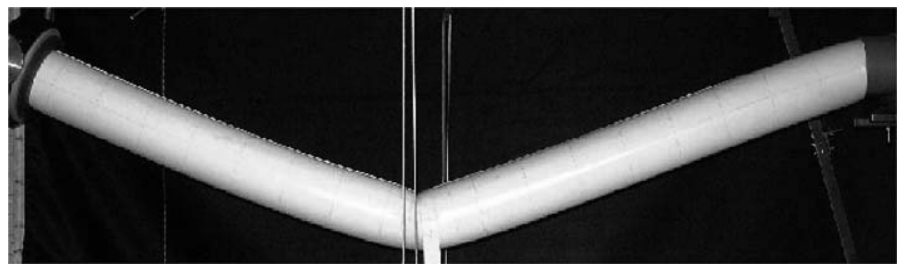

Fig. 3. Mechanism shape of an inflated beam in hyperstatic configuration.

deflections of a tube submitted to a given load $F$ depends on the values of the internal pressure. It is obvious that the pressure must be taken into account in the analytical solution of the values of the deflections. Fig. 3 shows the deformation pattern of the tube when the external load is near the collapse load of the tube. One can see that the shape is now similar to a mechanism.

These experimental results prove that the theory of the behaviour of inflatable tubes must be improved. The aim of the next section is to build the foundations of the mechanics of highly inflated tubes.

\section{Mechanics of inflatable tubes}

The mechanical strength of inflatable tubes is obtained by the use of equilibrium equations in the deformed state (to take into account the following forces due to the applied pressure), the constitutive laws of the fabrics and kinematical assumptions on the deformation pattern of the tube. The theory will be developed for a pressurized tube loaded by a concentrated force. The tube under pressure without any other loads is supposed to be the initial position. Fig. 4 defines the main notations: $v(x)$ is the deflection, $\alpha(x)$ is the rotation of the straight section, $N(x)$ is the normal resultant stress, $T(x)$ is the resultant shear stress and $M$ is the bending momentum. $R$ denotes the radius of the tube and $p$ is the internal pressure.

\subsection{Influence of the inflation pressure}

The pressure effects are replaced by forces normally applied to the internal area of the tube. They induce pre-stressing in the fabrics. They act as following forces and are considered as external forces. Two parts of the tube are concerned: the two boundary sections and the lateral walls (Fig. 5).

Two important assumptions allow us to take into account the forces due to internal pressure. The pressure is supposed to be uniform in the whole tube, and the cross-section is supposed to remain circular. The effects of the inflation pressure 


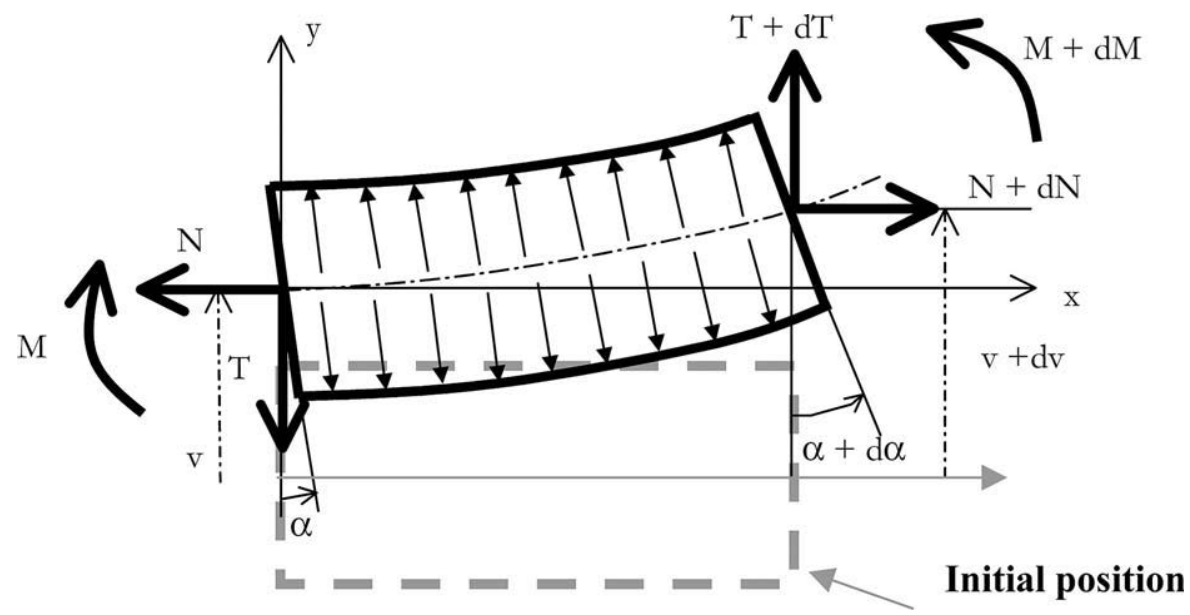

Fig. 4. Small element of the inflated tube.

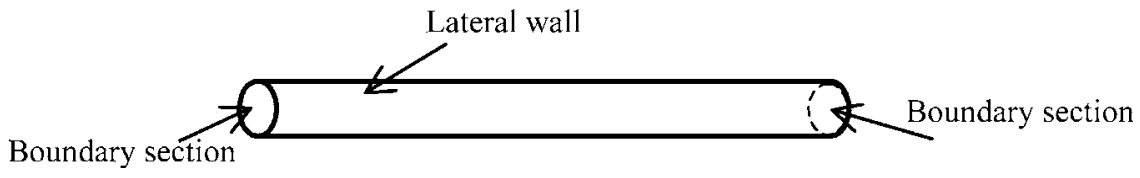

Fig. 5. Areas submitted to the inflation pressure.

on the lateral walls comes mainly from the rotation of the straight section which appears on an ellipse (Fig. 6). A distributed force density may then be defined by (Fig. 7):

$$
\mathrm{d} f(x)=p \mathrm{~d} S=p \pi R^{2} \mathrm{~d} \alpha=P \mathrm{~d} \alpha=P \frac{\mathrm{d} \alpha}{\mathrm{d} x} \mathrm{~d} x
$$

where $P=\pi R^{2}$ is the force due to the pressure on a boundary section.

\subsection{Governing equations}

Equilibrium equations are written for a tube's element with small length (Fig. 8).

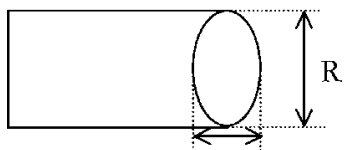

$\operatorname{Rd} \alpha$

Fig. 6. Ellipse submitted to pressure-upper view. 

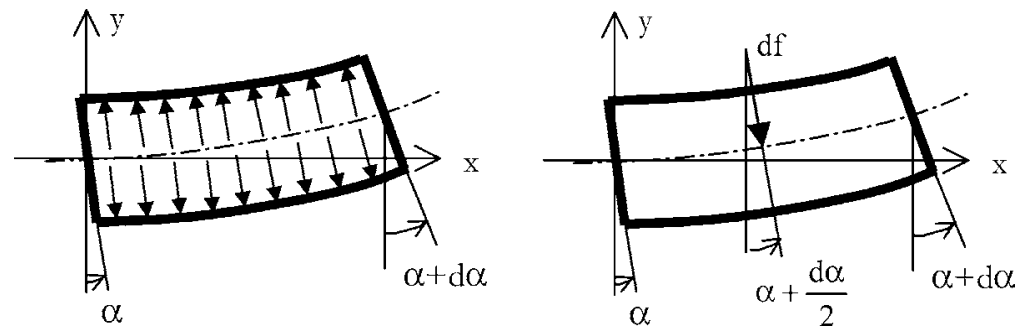

Fig. 7. Force density due to the inflation pressure applied on the lateral walls.

They are given by:

$$
\begin{aligned}
& \frac{\mathrm{d}}{\mathrm{d} x}\left(N-P \alpha^{2}\right)=0 \\
& \frac{\mathrm{d} T}{\mathrm{~d} x}-P \frac{\mathrm{d} \alpha}{\mathrm{d} x}=0 \\
& \frac{\mathrm{d} M}{\mathrm{~d} x}+T-N \frac{\mathrm{d} v}{\mathrm{~d} x}=0
\end{aligned}
$$

Timoshenko's beam theory must be used to describe the kinematics of the tube (Fig. 2). The cross-section is supposed to stay in a plane after deformation. $\alpha(x)$ may be different from the slope of the neutral fibre. The displacement of $G$ is supposed to satisfy the following relation:

$$
\vec{u}(G)=u(x) \vec{e}_{x}+v(x) \vec{e}_{y}
$$

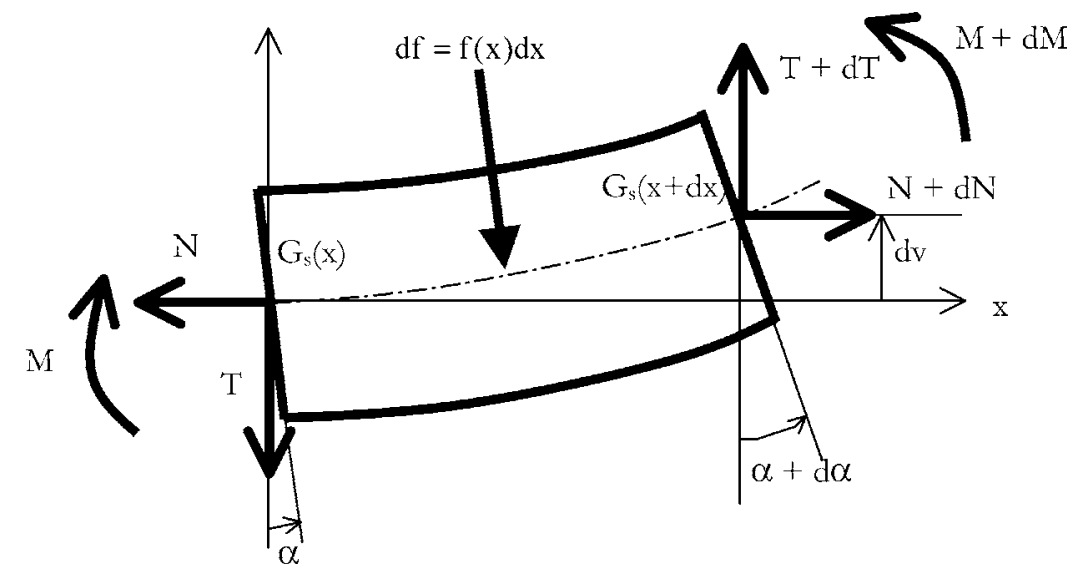

Fig. 8. Small length element of the inflated tube in deformed configuration. 


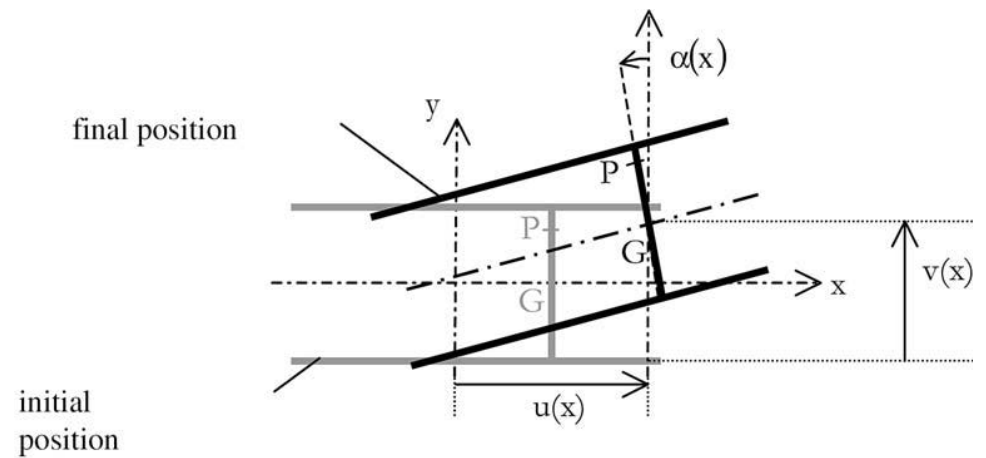

Fig. 9. Cross-section of the beam.

If $P$ is a point of the cross-section which is supposed to have a solid rigid behaviour, we have (Fig. 9):

$$
\vec{u}(P)=\vec{u}(G)+\vec{\Omega} \wedge \overrightarrow{P Q} \text { with } \vec{\Omega}=\alpha(x) \vec{e}_{z} \neq \frac{\mathrm{d} v}{\mathrm{~d} x} \vec{e}_{z}
$$

We will also suppose that the constitutive law of the fabric is isotropic. The constitutive laws of the tube are then given by two equations. Let us denote by $E^{*}$ [1] the tension membrane modulus (product of the Young modulus by the thickness $e$ of the fabric: $E^{*}=E e$ ) and by $G^{*}$ the shear membrane modulus $\left(G^{*}=G e\right) . K$ is the shear coefficient chosen equal to 0.5 according to Cowper's theory [8].

$$
\begin{aligned}
& T=K G^{*} S^{*}\left(\frac{\mathrm{d} v}{\mathrm{~d} x}-\alpha\right) \\
& M=E^{*} I^{*} \frac{\mathrm{d} \alpha}{\mathrm{d} x}
\end{aligned}
$$

\subsection{Boundary conditions}

They are also written in the deformed configuration. $F_{1}, \Gamma_{1}, F_{2}$ and $\Gamma_{2}$ denote the external forces and torques applied to the ends of the inflated tube (Fig. 10).

Boundary conditions must be accurately stated in order to take into account the following forces. Local equilibrium of nodes at the right and left boundaries of an inflated tube are described (Fig. 11) and give the following equations:

$$
\begin{aligned}
& N(0)=P ; \quad N(\ell)=P \\
& T(0)=-\left(F_{1}-P \alpha(0)\right) ; T(\ell)=F_{2}+P \alpha(\ell) \\
& M(0)=-\Gamma_{1} ; M(\ell)=\Gamma_{2}
\end{aligned}
$$

\subsection{Global equilibrium equations}

According to the governing equations and the boundary conditions, the global equilibrium equations are written in the deformed configuration. They lead to the 


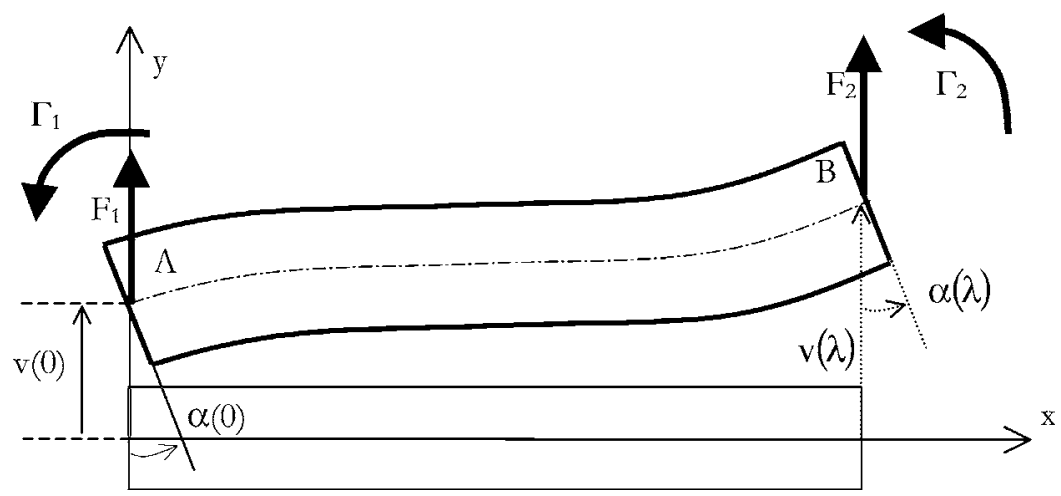

Fig. 10. Notation for the boundary's conditions.

following equations; the bending momentum equation is unusual

$$
\begin{aligned}
& F_{1}+F_{2}=0 \\
& \Gamma_{1}+\Gamma_{2}+\frac{K G^{*} S^{*}}{P+K G^{*} S^{*}} F_{2} \ell-\frac{P^{2}}{P+K G^{*} S^{*}}(v(\ell)-v(0))=0
\end{aligned}
$$

\subsection{Deflection of the inflated tube}

We will make the hypothesis that rotations remain small $(\alpha<<1)$; Eqs. (1) and (8) show that the normal resultant stress $N$ is constant:

$$
\begin{aligned}
& N(x)-P \alpha(x)^{2}=N(\ell)-P \alpha(\ell)^{2} \text { then } N(x)=P\left(1-\left(\alpha(x)^{2}-\alpha(\ell)^{2}\right)\right) \approx P \\
& N(x)=P
\end{aligned}
$$

The local equilibrium Eq. (3) becomes:

$$
\frac{\mathrm{d} M}{\mathrm{~d} x}+T-P \frac{\mathrm{d} v}{\mathrm{~d} x}=0
$$
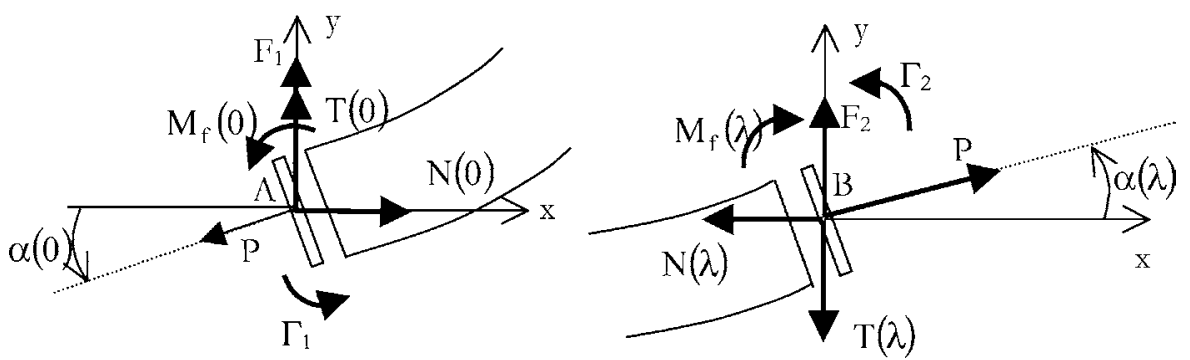

Fig. 11. Local equilibrium equations at the nodes of the inflated tube. 
By using Eq. (2), the shear resultant stress is

$$
T(x)=P \alpha(x)+F_{\mathrm{bc}}
$$

where $F_{\mathrm{bc}}$ is obtained by the boundary conditions.

The rotation of the section is therefore a solution of the differential equation:

$$
\frac{\mathrm{d}^{2} \alpha}{\mathrm{d} x^{2}}-\frac{P^{2}}{K E^{*} I^{*} G^{*} S^{*}} \alpha=\frac{F_{\mathrm{bc}}}{E^{*} I^{*}}\left(\frac{P-K G^{*} S^{*}}{K G^{*} S^{*}}\right)
$$

This solution is

$$
\alpha(x)=A \mathrm{e}^{\Omega x}+B \mathrm{e}^{-\Omega x}+\delta_{1}
$$

where $A$ and $B$ are constants to be calculated from the boundary conditions. $\Omega$ and $\delta_{1}$ are given by:

$$
\Omega=\frac{P}{\sqrt{K E^{*} I^{*} G^{*} S^{*}}} \text { and } \delta_{1}=\frac{\left(K G^{*} S^{*}-P\right)}{P^{2}} F_{\mathrm{bc}}
$$

The deflection of the inflated tube is then given by:

$$
v(x)=\frac{1}{\Omega}\left(\frac{K G^{*} S^{*}+P}{K G^{*} S^{*}}\right)\left(A \mathrm{e}^{\Omega \mathrm{x}}-B \mathrm{e}^{-\Omega \mathrm{x}}\right)+\frac{K G^{*} S^{*}}{K G^{*} S^{*}-P} \delta_{1} x+\delta_{2}
$$

Four constants are then needed in order to know the global behaviour of the inflated tube: $F_{\mathrm{bc}}, A, B$ and $\delta_{2}$.

3.6. Deflection of an inflated cantilever tube (Fig. 12)

The boundary conditions allow us to calculate the four unknown constants; they are:

$$
\alpha(0)=0, v(0)=0, T(\ell)=P \alpha(\ell)+F, M(\ell)=0
$$

The shear stress equation implies $F_{\mathrm{bc}}=F$ and then

$$
\delta_{1}=F \frac{\left(K G^{*} S^{*}-P\right)}{P^{2}}
$$

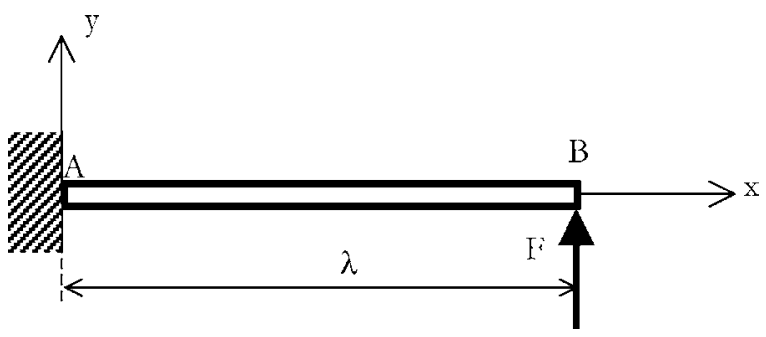

Fig. 12. Inflated cantilever tube. 
$v(0)=0$ leads to

$$
\delta_{2}=-\frac{1}{\Omega}\left(\frac{K G^{*} S^{*}+P}{K G^{*} S^{*}}\right)(A-B)
$$

$\alpha(0)=0$ gives

$$
A+B=\frac{F\left(P-K G^{*} S^{*}\right)}{P^{2}}
$$

$M(\ell)=0$ implies

$$
A \mathrm{e}^{\Omega \ell}=B \mathrm{e}^{-\Omega \ell}
$$

$A$ and $B$ are then calculated:

$$
A=F \frac{\left(P-K G^{*} S^{*}\right)}{P^{2}} \frac{\mathrm{e}^{-\Omega \ell}}{2 \operatorname{ch} \Omega \ell}
$$

and

$$
B=F \frac{\left(P-K G^{*} S^{*}\right)}{P^{2}} \frac{\mathrm{e}^{\Omega \ell}}{2 \operatorname{ch} \Omega \ell}
$$

The rotation and the deflection of the cantilever-inflated tube submitted to a concentrated load $F$ are finally given by:

$$
\left\{\begin{array}{l}
\alpha(x)=F \frac{\left(P-K G^{*} S^{*}\right)}{P^{2}} \frac{(\operatorname{ch} \Omega(\ell-x)-\operatorname{ch} \Omega \ell)}{\operatorname{ch} \Omega \ell}, \\
v(x)=F \frac{\left(K^{2} G^{2} S^{2}-P^{2}\right)}{K G^{*} S^{*} \Omega P^{2}} \frac{(\operatorname{sh} \Omega(\ell-x)-\operatorname{sh} \Omega \ell)}{\operatorname{ch} \Omega \ell}+\frac{K G^{*} S^{*}}{P^{2}} F x
\end{array}\right.
$$

One can see that the pressure appears in this formula by means of the terms $\Omega$, but that the beam and yarn effects dissociated in the case of inflatable panels [1] are here coupled by the shear stress influence.

\section{Comparisons between experimental and theoretical results}

Tubes have been tested by applying displacements with an extremely low strain rate. A load cell gives the applied load to the tube. The membrane modulus of Ferrari's fabric can be obtained from data given by the manufacturer. Unfortunately, we do not know exactly the conditions of the experiments used to define the materials properties. Moreover, we need the shear modulus, which is not given by the manufacturer. Finally, the fabrics have the ownership of viscous properties. All our experiments have been done after a time for which creep can be neglected. We have therefore made the choice to identify the material characteristics for one experiment on a tube with given radius, pressure and length between supports. The next results are relative to experiments conducted for different values of pressure and loads for this tube. The identification of the material properties gives:

$$
E^{*}=230,000 \mathrm{~Pa} \mathrm{~m}, \quad G^{*}=110,000 \mathrm{~Pa} \mathrm{~m}
$$



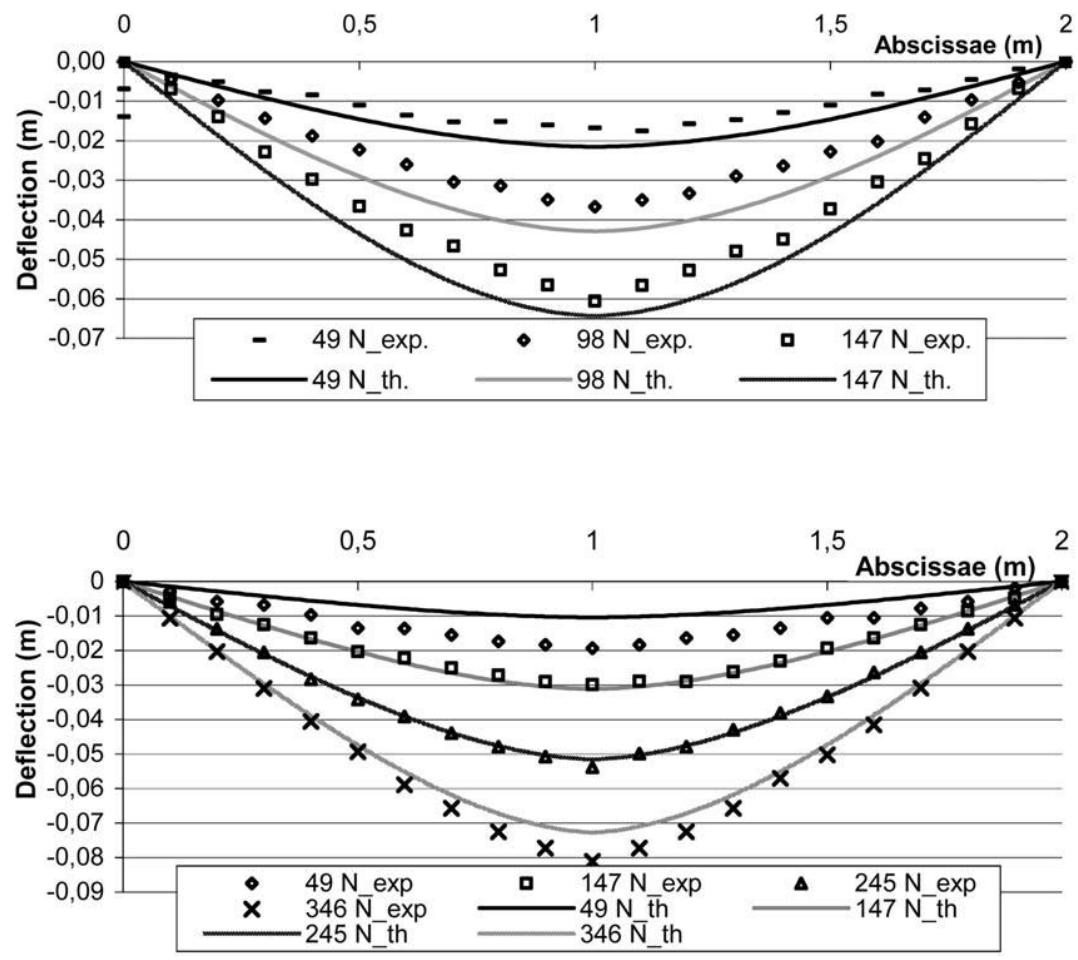

Figs. 13 and 14 show the comparisons between theoretical and experimental values of the deflections of one of the simply supported tubes for two values of pressure (100 and $300 \mathrm{kPa})$. One can see a good agreement between theoretical and experimental results.

\section{An inflatable fabric tube finite element}

The theory of inflatable fabric panels defined in [1] was the first attempt to built a finite element devoted to predicting inflatable panels deflections for hyperstatic configurations [6]. The compliance matrix of an inflatable panel in an isostatic configuration is symmetric and the usual theory of equilibrium finite elements has been used to construct the free displacement finite element able to solve the behaviour of inflated panels for hyperstatic configurations. Equations established in Section 3 lead to a non-symmetric compliance matrix and require another theory to build the tube finite element.

\subsection{A non-symmetrical compliance matrix}

Let us write the compliance matrix for the inflated tube defined in Fig. 15, submitted to a force $F_{2}$ and a torque $\Gamma_{2}$ at its free end. The only difference with the 


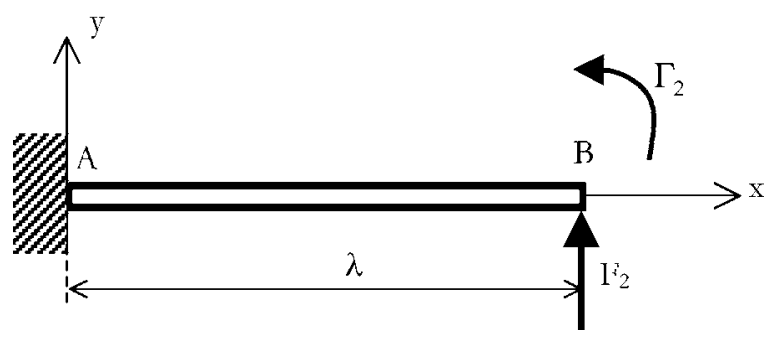

Fig. 15. Inflated tube in isostatic configuration.

analytical solution defined in Section 3 comes from the torque $\Gamma_{2}$. One boundary condition becomes:

$$
A \mathrm{e}^{\Omega \ell}-B^{-\Omega \ell}=\frac{\Gamma_{2}}{E I \Omega}
$$

The constants and $A$ and $B$ are therefore:

$$
\begin{aligned}
& A=\frac{1}{2 \operatorname{ch} \Omega \ell}\left[F_{2} \frac{\left(P-K G^{*} S^{*}\right)}{P^{2}} \mathrm{e}^{-\Omega \ell}+\Gamma_{2} \frac{1}{E^{*} I^{*} \Omega}\right] \\
& B=\frac{1}{2 \operatorname{ch} \Omega \ell}\left[F_{2} \frac{\left(P-K G^{*} S^{*}\right)}{P^{2}} \mathrm{e}^{\Omega \ell}-\Gamma_{2} \frac{1}{E^{*} I^{*} \Omega}\right]
\end{aligned}
$$

The analytical solution for the deflection of the tubes leads to this following nonsymmetrical compliance matrix:

$$
\begin{aligned}
\left\{\begin{array}{c}
v_{2} \\
\alpha_{2}
\end{array}\right\}= & {\left[\begin{array}{ll}
\frac{K G^{*} S^{*}}{P^{2}}\left(\ell-\frac{t h \Omega \ell}{\Omega}\right)+\frac{t h \Omega \ell}{K G S \Omega} & \left(\frac{K G^{*} S^{*}+P}{P^{2}}\right)\left(1-\frac{1}{c h \Omega \ell}\right) \\
\left(\frac{K G^{*} S^{*}-P}{P^{2}}\right)\left(1-\frac{1}{c h \Omega \ell}\right) & \frac{t h \Omega \ell}{E I \Omega}
\end{array}\right] } \\
& \times\left\{\begin{array}{c}
F_{2} \\
\Gamma_{2}
\end{array}\right\}
\end{aligned}
$$

The final finite element stiffness matrix cannot be built by means of the usual equilibrium finite element method and we will make use of the "left-quotient operation" to construct the stiffness matrix of the inflated tube element.

\subsection{The "left-quotient operation"}

The mathematical foundations of algebraic operations in structural mechanics which are used in the following can be found in [7,9]. The left-quotient operation allows the construction of exact finite elements even for non-symmetric problems [10] and must be used here. This section presents the main ideas of these algebraic operations. The left-quotient operation is used to replace the study of a continuous mechanical structure by the study of a discrete mechanical element (i.e. a finite element). Let us review the main results defining this "necessary" algebra. 
A mechanical structure $E$ may be presented as:

$$
E=\left(V,\langle. .,\rangle_{1}, \Phi\right)
$$

where $V$ and $\Phi$ are two linear spaces, $\langle. .,\rangle_{1}$ is a bilinear form which puts $V$ and $\Phi$ in duality and the mechanical law $L$ is a multiapplication which represents the equilibrium equations and the boundary conditions. In the studied case, $V$ is the generalized displacement space vector, $\Phi$ is the generalized loads space vector and the form $\langle. .,\rangle_{1}$ represents the work of the loads $\varphi \in \Phi$ in a displacement $v \in V$.

A sub-space $\Phi_{1}$ of the generalized loads space vector is defined by the nodal loads; $j$ is the canonical injection from $\Phi_{1}$ into $\Phi$, and $j^{\mathrm{T}}$ is its adjoint operator.

The equilibrium problem of $E$ submitted to the law $L$ is equivalent to the equilibrium problem of the left-quotient element $\left(V / \Phi_{1}^{0},\langle., .\rangle_{2}, \Phi_{1}\right)$ submitted to the load law $\left(j^{\mathrm{T}} \circ L^{-1} \circ j\right)^{-1} . V / \Phi_{1}^{0}$ is the left-quotient space (in fact the sub-space of the nodal displacements). The bilinear form $\langle., .\rangle_{2}$ represents the work of the nodal loads in the nodal displacements; $\Phi_{1}^{0}$ is the orthogonal of the sub-space $\Phi_{1}\langle. . .\rangle_{2}$ is then defined by:

$$
(\tilde{v}, \tilde{\varphi}) \in\left(V / \Phi_{1}^{0}, \Phi_{1}\right) \rightarrow\langle\tilde{v}, \tilde{\varphi}\rangle_{2}=\langle\bar{v}, j(\tilde{\varphi})\rangle_{1} \quad \forall \bar{v} \in j^{t^{-1}}(\tilde{v})
$$

The left-quotient diagram is given Fig. 16.

The finite element is built by solving the two following problems:

First problem: if the nodal loads $\tilde{\varphi} \in \Phi_{1}$ are given, find the nodal displacements $\tilde{v} \in V / \Phi_{1}^{0}$. The result $v \in V$ is obtained by solving the equilibrium equations with the Neumann boundary conditions defined by the law $L$. After that, the nodal displacements are deduced by $\tilde{v}=j^{t}(v)$. The result gives the compliance of the finite element: $\tilde{v}=\left(j^{t} \circ L^{-1} \circ j\right)(\tilde{\varphi})$.

Second problem: if the nodal displacements $\tilde{v} \in V / \Phi_{1}^{0}$ are given, find the nodal loads $\tilde{\varphi} \in \Phi_{1}$. The stiffness matrix is obtained by solving this inverse problem: $\tilde{\varphi}=\left(j^{t} \circ L^{-1} \circ j\right)^{-1}(\tilde{v})$.

\subsection{The stiffness matrix for the inflated tubes}

The aim of the operation is to replace the continuous problem by an equivalent discrete system (Fig. 17).

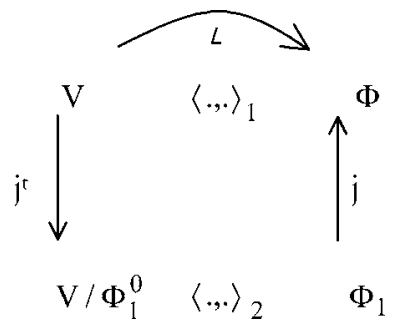

Fig. 16. Left-quotient operation diagram. 


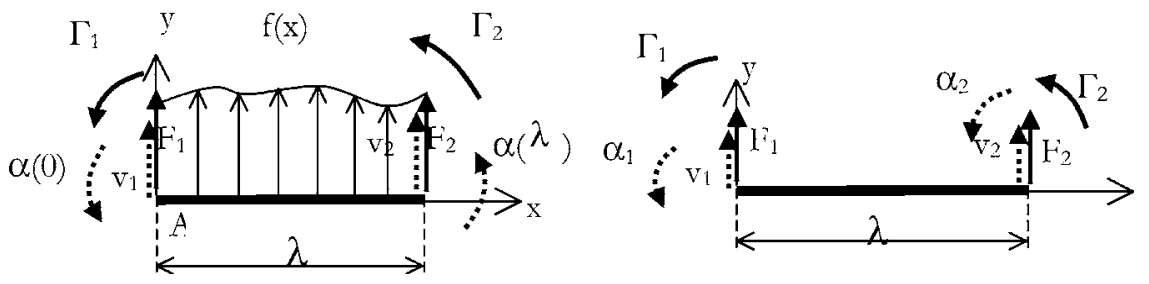

Fig. 17. Continuous and discrete problem.

The space vectors used here are defined by:

$$
V=C^{\infty}([0, \ell] ; R), \quad \Phi=C^{\infty}([0, \ell] ; R) \times R^{4}, \quad \Phi_{1}=R^{4}, \quad V / \Phi_{1}^{0}=R^{4}
$$

$\tilde{\varphi}$ represents the nodal loads $\left(F_{1}, \Gamma_{1}, F_{2}, \Gamma_{2}\right)$ and $\tilde{v}$ represents the nodal displacements $\left(v_{1}, \alpha_{1}, v_{2}, \alpha_{2}\right)$.

The solution of the first problem is given below. The analytical equations lead to the solutions (17) and (19):

$$
\left\{\begin{array}{l}
\alpha(x)=A \mathrm{e}^{\Omega x}+B \mathrm{e}^{-\Omega x}+\delta_{1} \\
v(x)=\frac{1}{\Omega}\left(1+\frac{P}{K G^{*} S^{*}}\right)\left(A \mathrm{e}^{\Omega x}-B \mathrm{e}^{-\Omega x}\right)-\frac{K G^{*} S^{*}}{P-K G^{*} S^{*}} \delta_{1} x+\delta_{2}
\end{array}\right.
$$

where four constants have to be determined.

The four boundary conditions are:

$$
\begin{aligned}
& -\left(F_{1}-P \alpha(0)\right)=K G^{*} S^{*}\left(\frac{\mathrm{d} v}{\mathrm{~d} x}(0)-\alpha(0)\right) \\
& -\Gamma_{1}=E^{*} I^{*} \frac{\mathrm{d} \alpha}{\mathrm{d} x}(0) \\
& \left(F_{2}+P \alpha(\ell)\right)=K G^{*} S^{*}\left(\frac{\mathrm{d} v}{\mathrm{~d} x}(\ell)-\alpha(\ell)\right) \\
& \Gamma_{2}=E^{*} I^{*} \frac{\mathrm{d} \alpha}{\mathrm{d} x}(\ell)
\end{aligned}
$$

In fact, Eq. (34) is equivalent to Eq. (36) and Eq. (35) leads to the same result as (37). The use of the four boundary conditions leads then to the following system where two independent constants $B$ and $\delta_{2}$ remain:

$$
\begin{aligned}
& \left\{V_{1}\right\}=[\underline{A}]\{X\}+[\underline{B}]\left\{\Phi_{1}\right\} \\
& \left\{V_{2}\right\}=[\underline{D}]\{X\}+\underline{[E]}\left\{\Phi_{1}\right\}
\end{aligned}
$$

where the vectors are defined as

$$
\left\{V_{1}\right\}=\left\{\begin{array}{c}
v_{1} \\
\alpha_{1}
\end{array}\right\}, \quad\{X\}=\left\{\begin{array}{c}
B \\
\delta_{2}
\end{array}\right\}, \quad\left\{\Phi_{1}\right\}=\left\{\begin{array}{c}
F_{1} \\
\Gamma_{1}
\end{array}\right\}, \quad\left\{\Phi_{2}\right\}=\left\{\begin{array}{c}
F_{2} \\
\Gamma_{2}
\end{array}\right\}
$$


and the matrixes are:

$$
\begin{array}{ll}
\underline{[A]}=\left[\begin{array}{ll}
1 & 0 \\
0 & 2
\end{array}\right] & {[\underline{B}]=\left[\begin{array}{ll}
0 & -\frac{\left(P+K G^{*} S^{*}\right)}{P^{2}} \\
\frac{\left(P-K G^{*} S^{*}\right)}{P^{2}} & \frac{-1}{E^{*} I^{*} \Omega}
\end{array}\right]} \\
{[\underline{D}]=\left[\begin{array}{ll}
1 & \frac{\left(P+K G^{*} S^{*}\right)}{K G^{*} S^{*} \Omega}\left(\mathrm{e}^{\Omega \ell}-\mathrm{e}^{-\Omega \ell}\right) \\
0 & \mathrm{e}^{\Omega \ell}+\mathrm{e}^{-\Omega \ell}
\end{array}\right]} & {[\underline{E}]=\left[\begin{array}{ll}
-\frac{K G^{*} S^{*}}{P^{2}} & -\frac{\left(P+K G^{*} S^{*}\right) \mathrm{e}^{\Omega \ell}}{P^{2}} \\
\frac{\left(P-K G^{*} S^{*}\right)}{P^{2}}-\frac{\mathrm{e}^{\Omega \ell}}{E^{*} I^{*} \Omega}
\end{array}\right]}
\end{array}
$$

The nodal displacements $\tilde{v}_{1} \in V / \Phi_{1}^{0}$ have therefore been found. They are defined nearly the rigid body modes. This fact explains the singularity of the equation set.

We have therefore obtained the compliance matrix of a free inflated beam.

The solution of the second problem is now displayed:Let us define $[\underline{L}]$ by $[\underline{L}]=\left([\underline{E}]-[\underline{D}][\underline{A}]^{-1}[\underline{B}]\right)$. Only the first part of the stiffness matrix can be calculated:

$$
\left\{\Phi_{1}\right\}=[\underline{L}]^{-1}\left\{V_{2}\right\}-[\underline{L}]^{-1}[\underline{D}][\underline{A}]^{-1}\left\{V_{1}\right\}
$$

The necessary condition for existence of solutions to the last problem gives the two missing equations which are Eqs. (11) and (12). They can be written as:

$$
\left\{\Phi_{2}\right\}=[\underline{H}]\left\{\Phi_{1}\right\}-\{\underline{G}\}\left\{V_{2}\right\}+\{\underline{G}\}\left\{V_{1}\right\}
$$

where

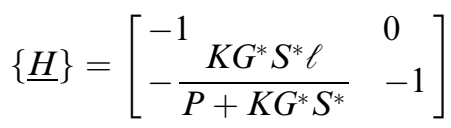

and

$$
\{\underline{G}\}=\left[\begin{array}{ll}
0 & 0 \\
\frac{P^{2}}{P+K G^{*} S^{*}} & 0
\end{array}\right]
$$

The global static equilibrium equations then give:

$$
\left\{\Phi_{2}\right\}=-\left([\underline{H}][\underline{L}]^{-1}[\underline{D}][\underline{A}]^{-1}+[\underline{G}]\right)\left\{V_{1}\right\}+\left([\underline{G}]+[\underline{H}][\underline{L}]^{-1}\right)\left\{V_{2}\right\}
$$

and finally, the stiffness matrix is obtained (it is $\left.\tilde{\varphi}=\left(j^{t} \circ L^{-1} \circ j\right)^{-1}(\tilde{v})\right)$

$$
\left\{\begin{array}{l}
\left\{\Phi_{1}\right\} \\
\left\{\Phi_{2}\right\}
\end{array}\right\}=\left[\begin{array}{ll}
-[\underline{L}]^{-1}[\underline{D}][(\underline{A})]^{-1} & {[\underline{L}]^{-1}} \\
-\left([\underline{H}][\underline{L}]^{-1}[\underline{D}][\underline{A}]^{-1}+[\underline{G}]\right) & {[\underline{G}]+[\underline{H}][\underline{L}]^{-1}}
\end{array}\right]\left\{\begin{array}{l}
\left\{V_{1}\right\} \\
\left\{V_{2}\right\}
\end{array}\right\}
$$


The stiffness matrix is then written:

$$
R=\left[\begin{array}{llll}
A_{1} & A_{3} & -A_{1} & A_{3} \\
A_{4} & A_{2} & -A_{4} & A_{5} \\
-A_{1} & -A_{3} & A_{1} & -A_{3} \\
A_{4} & A_{5} & -A_{4} & A_{2}
\end{array}\right]
$$

which is a non-symmetrical matrix. The matrix coefficients are tedious to write, but they explicitly express the inflation pressure:

$$
\begin{aligned}
& A_{1}=\frac{P^{2} K G^{*}+{ }^{*} \Omega\left(1+\mathrm{e}^{\Omega \ell}\right)}{2\left(\left(K G^{*} S^{*}\right)^{2}-P^{2}\right)\left(1-\mathrm{e}^{\Omega \ell}\right)+\left(K G^{*} S^{*}\right)^{2} \Omega \ell\left(1+\mathrm{e}^{\Omega \ell}\right)} \\
& A_{2}=\frac{E^{*} I^{*} \Omega\left(P^{2}-\left(K G^{*} S^{*}\right)^{2}\right)\left(\mathrm{e}^{2 \Omega \ell}-1\right)+K G^{*} S^{*} \ell P^{2}\left(1+\mathrm{e}^{2 \Omega \ell}\right)}{2 P^{2}\left(1-2 \mathrm{e}^{\Omega \ell}+\mathrm{e}^{2 \Omega \ell}\right)+2\left(K G^{*} S^{*}\right)^{2}\left(2 \mathrm{e}^{\Omega \ell}-1-\mathrm{e}^{2 \Omega \ell}\right)+\left(K G^{*} S^{*}\right)^{2} \ell \Omega\left(\mathrm{e}^{2 \Omega \ell}-1\right)} \\
& A_{3}=\frac{-P^{2}\left(P+K G^{*} S^{*}\right)\left(1-\mathrm{e}^{\Omega \ell}\right)}{2\left(\left(K G^{*} S^{*}\right)^{2}-P^{2}\right)\left(1-\mathrm{e}^{\Omega \ell}\right)+\left(K G^{*} S^{*}\right)^{2} \Omega \ell\left(1+\mathrm{e}^{\Omega \ell}\right)} \\
& A_{4}=\frac{P^{2}\left(P-K G^{*} S^{*}\right)\left(1-\mathrm{e}^{\Omega \ell}\right)}{2\left(\left(K G^{*} S^{*}\right)^{2}-P^{2}\right)\left(1-\mathrm{e}^{\Omega \ell}\right)+\left(K G^{*} S^{*}\right)^{2} \Omega \ell\left(1+\mathrm{e}^{\Omega \ell}\right)} \\
& A_{5}=-\frac{E^{*} I^{*} \Omega\left(P^{2}-\left(K G^{*} S^{*}\right)^{2}\right)\left(\mathrm{e}^{2 \Omega \ell}-1\right)+2 K G^{*} S^{*} \ell P^{2} \mathrm{e}^{\Omega \ell}}{2 P^{2}\left(1-2 \mathrm{e}^{\Omega \ell}+\mathrm{e}^{2 \Omega \ell}\right)+2\left(K G^{*} S^{*}\right)^{2}\left(2 \mathrm{e}^{\Omega \ell}-1-\mathrm{e}^{2 \Omega \ell}\right)+\left(K G^{*} S^{*}\right)^{2} \ell \Omega\left(\mathrm{e}^{2 \Omega \ell}-1\right)}
\end{aligned}
$$

\section{Comparisons between experimental and finite element results}

This stiffness matrix (44) devoted to the study of inflated tubes has been included in a finite element software in order to solve hyperstatic problems. Two comparisons between experimental and numerical results are presented (Figs. 18 and 19).

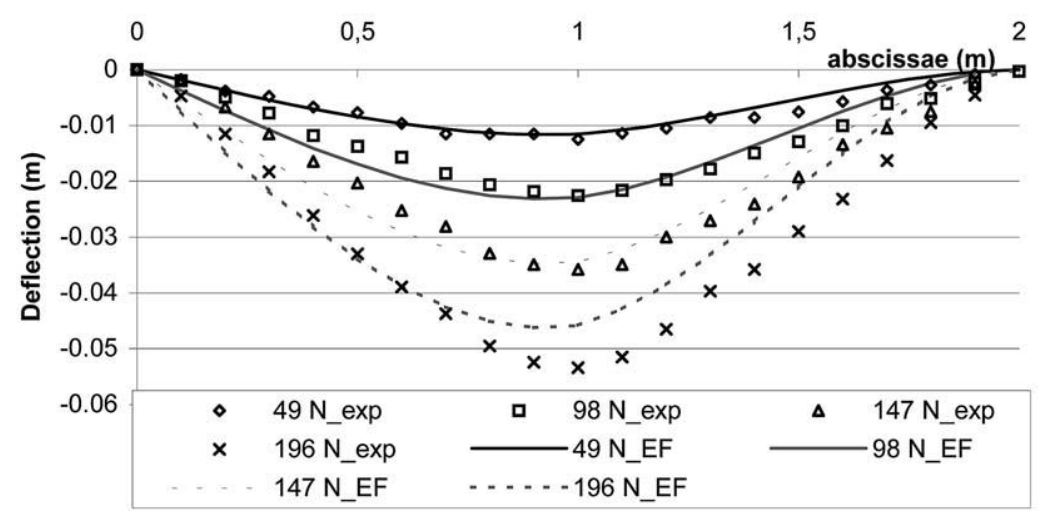

Fig. 18. Deflection of an inflated tube- $-100 \mathrm{kPa}$. 


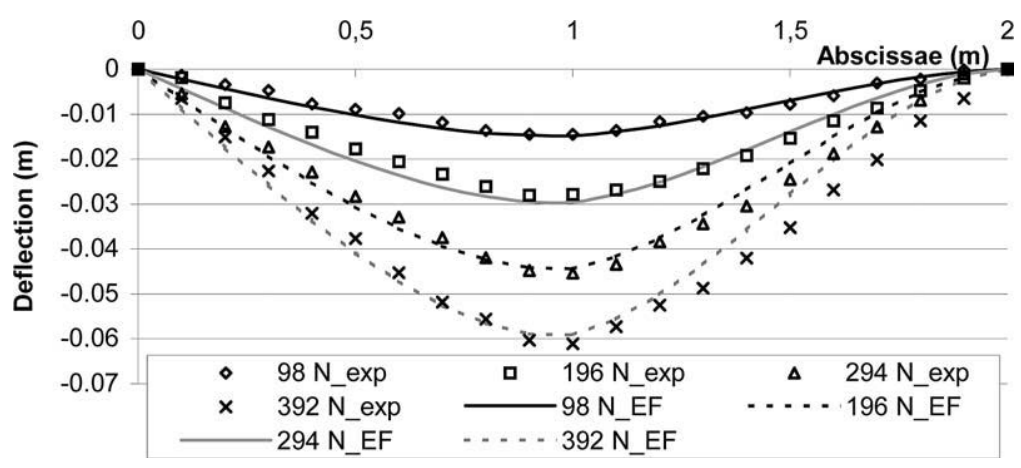

Fig. 19. Deflection of an inflated tube $-300 \mathrm{kPa}$.

The shape of the tube is well-defined, and the difference between experimental results and numerical ones is less than $15 \%$.

\section{Conclusion}

Inflated tubes play an important role in the design of inflated structures and their behaviour has to be understood. Modern textile materials with high mechanical characteristics allow us to foresee the use of highly inflated beams in building. The main results of the paper are relative to the deflections of inflatable tubes under bending loads. The real problem is very complicated to study because of the material and the geometrical non-linearities. We show here that with a minimum of assumptions, we are able to give simple analytical formula to estimate the deflections of inflatable tubes submitted to bending loads. Experiments have shown the influence of the shear stress and Timoshenko's beam theory is used to describe the kinematics of the tubes because the cross-sections of the panels do not satisfy the usual Bernoulli beam theory. The inflation pressure appears in the solution and is taken into account by writing the equilibrium equations in the deformed state. Comparisons between experimental and analytical results on the behaviour of simply supported inflatable tubes prove the accuracy of this theory. Analytical solutions show that the compliance matrix of a pressurized cantilever beam is not symmetrical, which was not the case for inflatable panels. The usual equilibrium finite element theory used in [6] cannot simply be applied to these kinds of problems. We have therefore chosen to use algebraic operations to derive a new finite element for the tubes which has, moreover, the property of giving an exact solution, and allows the study of tubes in hyperstatic configurations. The result gives a non-symmetrical stiffness matrix. This new finite element has been implemented in a finite element software. Comparisons between experimental and numerical results are shown. Numerical results are close to experimental ones. 


\section{References}

[1] Wielgosz C, Thomas JC. Deflections of inflatable fabric panels at high pressure. Thin-Walled Structures 2002;40:523-36.

[2] Comer RL, Levy S. Deflections of an inflated circular cylindrical cantilever beam. AIAA Journal 1963;1(7):1652-5.

[3] Fichter WB. A theory for inflated thin-wall cylindrical beams. NASA TN D-3466. 1966.

[4] Main A, Peterson SW, Strauss AM. Load-deflection behaviour of space-based inflatable fabric beams. Journal of Aerospace Engineering 1994;2(7):225-38.

[5] Wielgosz C, Thomas JC, Casari P. Strength of inflatable fabric beams at high pressure. 43rd AIAA/ASME/ASCE/AHS Structures, Structural Dynamics, and Materials Conference, Denver. 2002, p. 1292.

[6] Wielgosz C, Thomas JC. An inflatable fabric beam finite element. Communications in Numerical Methods in Engineering 2003;19 (in press).

[7] Nayroles B. Opérations algébriques en mécanique des structures. CRAS 1971;273(A):1075-8.

[8] Cowper GR. The shear coefficient in Timoshenko's beam theory. Journal of Applied Mechanics 1967;33:335-40.

[9] Wielgosz C, Débordes O. Exact or quasi-exact numerical results for two-dimensional harmonic problems. European Journal of Mechanics, A/Solids 1990;9:453-76.

[10] Wielgosz C. Informations exactes données par des méthodes d'éléments finis en mécanique. Journal de Mecanique Theorique et Appliquée 1982;1(2):323-9. 